\title{
Forepaw Sensorimotor Deprivation in Early Life Leads to the Impairments on Spatial Memory and Synaptic Plasticity in Rats
}

\author{
Yuanyuan Zhang, ${ }^{1}$ Fei Li, ${ }^{2}$ Xiaohua Cao, ${ }^{3}$ Xingming Jin, ${ }^{2}$ Chonghuai Yan, ${ }^{1}$ \\ Ying Tian, ${ }^{1,4}$ and Xiaoming Shen ${ }^{1}$ \\ ${ }^{1}$ Shanghai Key Laboratory of Children's Environmental Health, Shanghai Institute for Pediatric Research, Xin Hua Hospital, \\ Shanghai Jiao Tong University School of Medicine, Shanghai 200092, China \\ ${ }^{2}$ Department of Developmental and Behavioral Pediatrics of Shanghai Children's Medical Center, \\ Shanghai Jiao Tong University School of Medicine, Shanghai 200127, China \\ ${ }^{3}$ Shanghai Institute of Brain Functional Genomics, The Key Laboratories of Ministry of Education (MOE) of China and Science and \\ Technology Commission of Shanghai Municipality (STCSM), East China Normal University, Shanghai 200062, China \\ ${ }^{4}$ Department of Environmental Health, Shanghai Jiao Tong University School of Medicine, Shanghai 200025, China
}

Correspondence should be addressed to Xiaoming Shen, xmshen@shsmu.edu.cn

Received 27 March 2009; Accepted 8 October 2009

Recommended by Kanury Rao

To investigate the influence of forepaw sensorimotor deprivation on memory and synaptic plasticity, Sprague-Dawley rats were divided into two groups: a sham-operated group and a group deprived of forepaw sensorimotor function by microsurgical operation at postnatal day 13 (PN13). Behavioral and electrophysiological studies were performed at PN25, PN35, PN45, and PN60. Open field test was used to assess the spontaneous locomotor activity. Morris water maze was used to evaluate spatial reference learning and memory. The long-term potentiation (LTP) in the medial perforant path—dentate gyrus (MPP-DG) pathway was examined with hippocampal slices. We found that forepaw sensorimotor deprivation did not affect spontaneous activity of the rats. However, spatial reference learning and memory were significantly impaired in their early life (PN25, PN35, and PN45). In accordance with the behavior results, LTP in MPP-DG pathway was significantly suppressed in their early life. These data demonstrated that forepaw sensorimotor deprivation led to the impairments on spatial memory via inducing pronounced deficits in the MPP-DG pathway to exhibit LTP, one of the major cellular mechanisms underlying learning and memory.

Copyright ( 2009 Yuanyuan Zhang et al. This is an open access article distributed under the Creative Commons Attribution License, which permits unrestricted use, distribution, and reproduction in any medium, provided the original work is properly cited.

\section{Introduction}

Over the past few years, there has been an increasing interest in the relationship between cognitive and sensorimotor performances during the developmental process in children. It is widely accepted that cognitive development should not be isolated from sensorimotor function. In other words, intelligence and achievement are established on the precondition of full development of sensorimotor function. Furthermore, high-level thinking and behavior depend on the integration of sensory input and action response to accomplish. However, there was little experimental evidence supporting this idea.
Cognitive processes were originally believed to be independent of the motor processes. In the middle of last century, Piaget pointed out that cognitive and motor processes were not separate entities, in fact, cognitive development relies on motor functioning [1]. To further understand the role of cognition in evolution, Churchland suggested its origin in sensorimotor control and hypothesized the existence of a continuum of higher sensorimotor functions and higher cognitive functions [2, 3]. Moreover, specific brain structures and neurotransmitters have been proved to be involved in both cognitive and motor performances. Cognitive performance related to behavior planning and executive functioning develop in early life period, when 
motor processes, such as movement control and visuomotor coordination, develop rapidly. On the other hand, the children with cognitive deficits often show delayed motor development [4], and inadequate motor performance is thought to be a marker of possible academic problems [5].

Learning and memory are ones of the most important components of cognitive function, and it could well predict the academic achievement in the future. When cognitive function is impaired, learning and memory are most commonly involved. Furthermore, neural plasticity, which is indicated by LTP, is considered to be the electrophysiological basis for learning and memory. Evidence from animal experiments has shown that forced or voluntary physical activity in a running wheel promotes DG neurogenesis in rodents, facilitates induction of LTP, and enhances learning and memory performance $[6,7]$. Furthermore, this exercise can rescue deficits in spatial memory and LTP in rats exposed to detrimental factors such as prenatal ethanol exposure [8]. Taking together, these data suggest that gross motor training such as running is beneficial to synaptic plasticity and cognitive development which raise the question whether specific fine motor training can also augment brain plasticity and promote cognitive development.

To address this question, we went through several wellestablished models. Most models for sensorimotor training at present are based on calorie restriction, after which rats are motivated by the wish for acquiring food to develop precise manipulation of forepaw and digit movement $[9$, 10]. However, calorie restriction in the early life period impedes the growth and development of rats in itself. Kudryashov and Kudryashova therefore started a forelimb denervation procedure in rats for studying the effects of sensory impairment on synaptic transmission and plasticity [11]. Because median and ulnar nerves are mixed nerves responsible for forepaw sensory and movement, the latter refers to the strength and coordination of the grip [12-14]. In this study we used a similar model by removal of both median and ulnar nerves at the ligament carpi transversum of both forelimbs.

Moreover, during the rehabilitation after ischemic injury, task-specific rehabilitative therapy such as reach training has been demonstrated to facilitate functional reorganization within the intact sensorimotor cortex and ameliorate sensorimotor function of the impaired forelimb and digits [10]. However, whether cognitive function also improved is not explored. We previously found [15] that sensorimotor deprivation induced by transection of the forepaw median and ulnar nerves at PN18 led to significant impairments on spatial memory and synaptic plasticity in the schaffer-CA1 pathway. With similar surgery, Kudryashov and Kudryashova reported median nerve transection significantly alters longterm potentiation (LTP) in this pathway in hippocampus [11]. It is not known whether synaptic plasticity in MPPDG pathway is also affected and whether earlier sensorimotor deprivation may produce more severe impairment.

Thus, the aim of this study was to investigate the influence of early forepaw sensorimotor deprivation in young rats on spatial reference memory and synaptic plasticity in MPPDG pathway.

\section{Materials and Methods}

2.1. Animal Care and Surgery. 13-day-old, specific-pathogen free Sprague-Dawley rats were used for this study (Shanghai Laboratory Animal Center, Shanghai, China). Littermate rats paired by weights were assigned at random into four groups. Half of the rats in each group received sensorimotor deprivation via transection treatment $(\mathrm{T})$ of the median and ulnar nerves of bilateral forepaws as previously described $[11,15]$, by removal of $1.5 \sim 2 \mathrm{~mm}$ of the median and ulnar nerves at the ligament carpi transversum of both forelimbs. The rest served as controls (C) and were sham operated. The pups were weaned at PN21, after which they were group housed according to litter and sex in clear polycarbonate cages, with ad libitum access to standard rat chow and water. The room temperature was maintained at $22-26^{\circ} \mathrm{C}, 40-70 \%$ humidity; the rats were kept in a 12-hour light/dark cycle (lights on from 07 : $00 \mathrm{AM}$ ). Spatial reference learning and memory were assessed by Morris water maze test in each group of rats beginning at one of the following times (rat age and $\mathrm{C}$ and $\mathrm{T}$ subdesignations noted in parentheses): 12 days (PN25; C25 and T25), 22 days (PN35; C35 and T35), 32 days (PN45; C45 and T45), and 47 days after surgery (PN60; C60 and $\mathrm{T} 60$ ).

All experiments were performed in a blind manner during the light phase of the cycle. All the experimental procedures involving the use of animals were approved by the Ethical Committee for Experimental Animal Usage of the Shanghai Jiao Tong University School of Medicine and the East China Normal University.

2.2. Behavioral Tests. All of the behavioral tests were conducted in the well-designed behavioral rooms, which were sound-proof, located in the animal facility. The arena for behavioral tests was protected by a black curtain, to ensure elimination of any other possible visual clues or interference.

2.2.1. Open Field Locomotor Test. Rats were individually placed in the center of an opaque plexi cage equipped with the photobeam sensor rings that automatically detected and quantified their activities and were allowed to explore the environment for 15 minutes. Frequency of horizontal movement (FP_moves), locomotion time (FP_move time), and total distance traveled (FP_distance) were measured using a Tru-scan DigBehv-locomotion Activity Video Analysis System (Coulbourn instruments, USA). These indices were used to analyze locomotor activity and exploratory behavior.

2.2.2. Morris Water Maze Test. The water maze test was performed on the Poly Track video tracking system (San Diego Instruments). A circle pool (160 cm in diameter) was used and the platform $(12 \mathrm{~cm}$ in diameter) was placed in the centre of any quadrant of the pool and submerged $1 \mathrm{~cm}$ underneath the water surface. The training protocol consisted of four to six sessions, one session (three trials) per day [16, 17]. The starting quadrant was randomly arranged for each animal and each session (three trials) 
contained a starting position from all but the platform quadrants. Each trial began with the rat being placed in the pool and released facing the side wall at one of the three starting positions. On each trial, the rat was allowed to swim until it found and remained on the platform for 15 seconds. The escape latency (time to reach the platform) was automatically recorded. If the rat did not find the platform within 90 seconds, it was guided to the platform by the experimenter and allowed to stay on the platform for 15 seconds. The number of training sessions was determined by when group $\mathrm{C}$ remembers the position of the platform. Once group $\mathrm{C}$ found the platform within 15 seconds, the probe test was performed the next day. During the probe test, the platform was removed and the rats were allowed to swim in the pool for 90 seconds. The time spent in each quadrant during searching was recorded, and targeted quadrant time \% (targeted quadrant swimming time relative to total swimming time) were automatically calculated. All tests were carried out during the light cycle, between $08: 00$ and 14:00. The rats were tested in pseudorandom order. Escape latency during the training sessions and targeted quadrant time \% during the probe test were used to evaluate their performances in this task. Swimming speed across trials was also automatically analyzed, to exclude the possibility that the dependent variable measure, latency to the platform, might be influenced by extraneous variables such as fitness.

\subsection{Electrophysiological Recordings and LTP Induction. Hip-} pocampal slices were prepared as previously described $[18,19]$. Briefly, the rats were anesthetized with sodium pentobarbital (2\%) and decapitated. The brains were rapidly removed and the hippocampi were dissected in icecold oxygenated $\left(95 \% \mathrm{O}_{2}\right.$ and $\left.5 \% \mathrm{CO}_{2}\right)$ artificial cerebrospinal fluid (ACSF). The ACSF contained (in $\mathrm{mM}$ ): $120 \mathrm{NaCl}$, $2.5 \mathrm{KCl}, 1 \mathrm{NaH}_{2} \mathrm{PO} 4 \cdot 2 \mathrm{H} 2 \mathrm{O}, 1.3 \mathrm{MgSO}_{4}, 2.5 \mathrm{CaCl}_{2}, 26$ $\mathrm{NaHCO}_{3}$, and 10 D-glucose, $\mathrm{pH}$ 7.3-7.4, bubbled with $95 \% \mathrm{O}_{2}$ and $5 \% \mathrm{CO}_{2}$. Approximately $400 \mu \mathrm{m}$ transverse slices were prepared on a tissue chopper and then incubated in a recovery chamber filled with the oxygenated ACSF. Slices were allowed to recover for at least 1.5 hours before recordings were attempted. For electrophysiological recordings, the slices were transferred into a perfusion chamber continuously superfused with ACSF at a rate of $2.5 \mathrm{~mL} / \mathrm{min}$ and maintained at $30^{\circ} \mathrm{C}$ while recording. All experiments were done on slices maintained in vitro for 2-8 hours.

For extracellular stimulation of the MPP, a monopolar tungsten electrode was placed in the middle molecular layer of the dentate gyrus. Extracellular field EPSPs were recorded with a glass microelectrode (2-10 $\mathrm{M} \Omega$ resistance) filled with pontamine sky blue and situated in the middle molecular layer of the dentate gyrus. Responses were evoked using single pulse stimuli of a fixed duration ( 0.1 millisecond), delivered at 30-second intervals. The optimal recording location for each animal was determined as the point at which the largest response could be obtained with a minimal amount of current being used. Only those slices which produced the field excitatory postsynaptic potentials (fEPSPs) of $1 \mathrm{mV}$ or higher in amplitude were accepted for experiments. Medial pathway responses were confirmed on the basis of depression of field EPSPs elicited with paired pulses spaced 40-100 milliseconds apart [20-22]. The stimulation intensity that produced $\sim 30 \%$ of maximal evoked amplitude was chosen for test pulse and tetanic stimulation. Test stimuli ( 0.1 millisecond pulse width) were delivered every 30 seconds before tetanization. Once a stable baseline recording was obtained, LTP was induced by modifying a protocol (high-frequency stimulation: 2 trains, 500 milliseconds each, $100 \mathrm{~Hz}$ within the train, repeated every 30 seconds) described by Snyder et al. [23]; the stimulus strength during tetanization was increased by doubling the pulse width to 0.2 millisecond. Bicuculline $(10 \mu \mathrm{m} / \mathrm{L})$ was applied to block GABAergic inhibition. The slope of the rising phase of the field EPSPs was used to determine alterations in the level of synaptic efficacy [21,22]. Following the administration of the conditioning stimuli, single pulse stimuli were again administered for a minimum period of 45 minutes. The preferred measure of response size, fEPSP slope data were presented as the mean percent change from the preconditioning baseline \pm SEM. And data during the last 10 minutes of recording were averaged to compare LTP induction with different treatment.

2.4. Statistical Analysis. Values of all variables are presented as means \pm SEM. Analysis of variance (One-way ANOVA and Two-way ANOVA) and posthoc Dunnett's tests [18] were used to determine the effects of different treatments. All analyses were conducted using SPSS statistical software version 13.0 (SPSS Inc., Chicago, IL). Statistical significance was set at $P<.05$.

\section{Results}

\subsection{Behavioural Performances}

3.1.1. Open Field Test-Locomotor Activity. To determine whether forepaw sensorimotor deprivation affected spontaneous locomotor activity in rats, three indices of horizontal movement (FP_moves, FP_move time, FP_distance) over a 15-minute period were used to assess locomotor activity in group $\mathrm{T}$ and group $\mathrm{C}$ rats. There was no significant difference in any of the above measures between these two groups at each time point (PN25, PN35, PN45, PN60) $(P>.05$; Figure 1$)$. These results indicated that forepaw sensorimotor deprivation had neither effects on locomotion nor exploratory behavior of the young rats.

\subsubsection{Morris Water Maze-Spatial Reference Learning and} Memory. To assess spatial reference learning and memory, we performed Morris water maze experiment. In this experiment, both groups of rats were trained on four to six sessions. Both groups of rats were able to learn the task, as demonstrated by the decreased escape latency to find the platform during training sessions. However, the sensorimotor deprived rats (Group T) displayed a slower decrease in their escape latency compared to their shamoperated control (Group C) counterparts at early time point. 


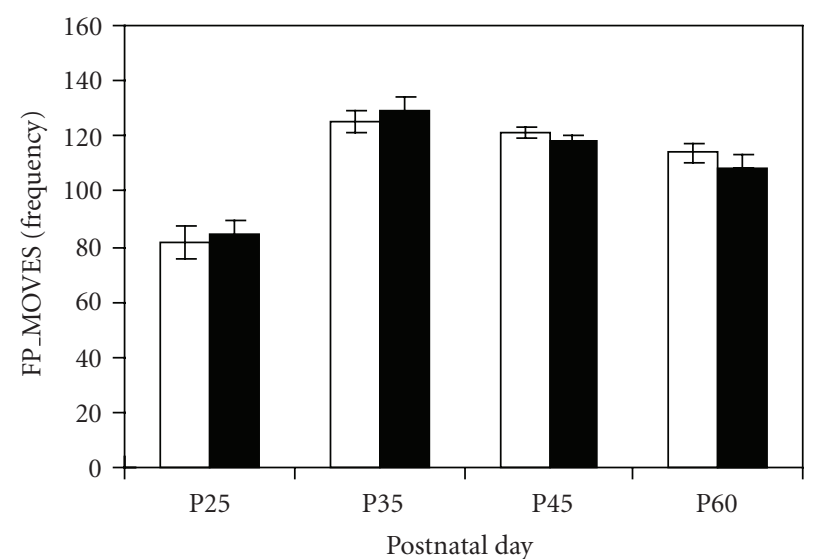

Control

$\square$ Treatment

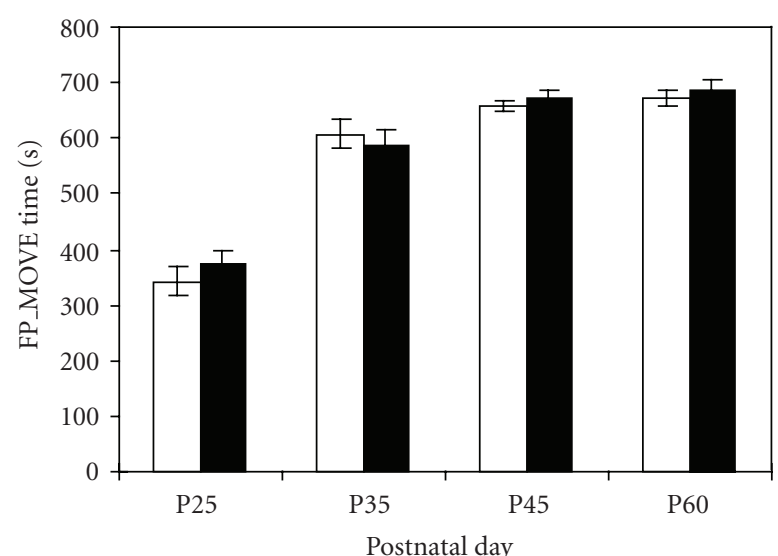

$\square$ Control

Treatment

(a)

(b)

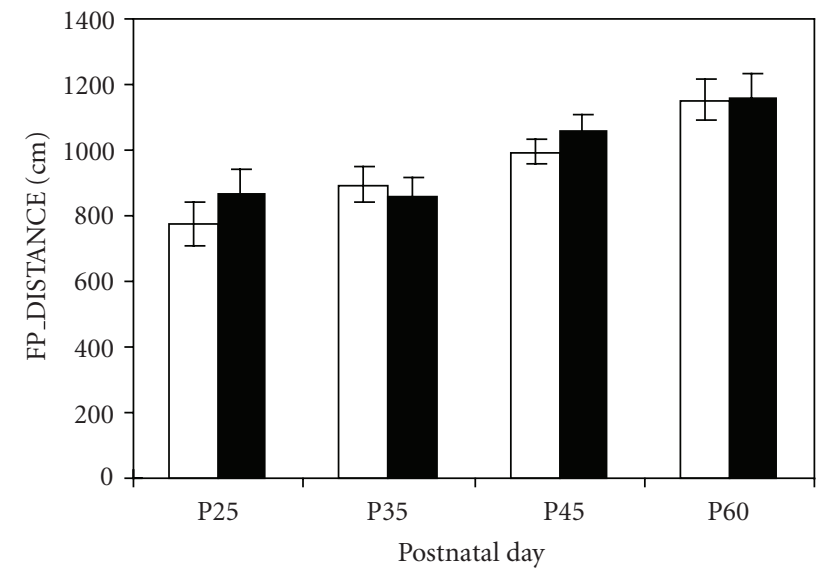

$\square$ Control

Treatment

(c)

FIGURE 1: Open field test performance in forepaw sensorimotor deprived group (treatment) and sham-operated control group (control). Forepaw sensorimotor deprivation did not affect spontaneous locomotor activity of the rats at PN25, PN35, PN45, and PN60 $(P>.05)$. The Three indices (a) FP_moves, (b) FP_move time, and (c) FP_distance were shown as above.

Furthermore, in the probe test, Group T spent less amount of time in searching the targeted quadrant.

At PN25, although all the rats started with the same level of performance at the beginning, Group T displayed a slower decrease in their escape latency. Two-way ANOVA revealed a significant difference in escape latency between Group $\mathrm{T}$ and Group C $(F=6.99, P<.01)$. In addition, a posthoc analysis by using Dunnett's test indicated that a significant difference in escape latency was found at the third $(P<.05)$ and fourth sessions $(P<.05)$ between these two groups (Figure $2(\mathrm{a}))$. By the third session, group $\mathrm{T}$ took approximately 46 seconds to find the platform, whereas group $\mathrm{C}$ took only an average of 32 seconds. In the following fourth session, group $\mathrm{T}$ took approximately 43 seconds to find the platform, whereas group $C$ took only an average of 28 seconds. In the probe test conducted after the final training session, group $\mathrm{T}$ spent significantly less amount of time in searching the targeted quadrant than group C $(34.13 \pm 2.65 \%$ versus $42.35 \pm 2.06 \%$; $F=6.09, P<.05$, Figure $2(\mathrm{f}))$.

Similarly, at PN35, Group T displayed a slower decrease in their escape latency $(F=4.25, P<.05)$. In the second session, group $\mathrm{T}$ took approximately 37 seconds to find the platform, whereas group $\mathrm{C}$ showed a significantly shorter escape latency (24.12 seconds; $P<.05$, Figure $2(\mathrm{~b}))$. In the probe test, group $\mathrm{T}$ spent significantly less amount of time in searching the targeted quadrant than group C $(23.86 \pm 2.15 \%$ versus $30.60 \pm 1.71 \% ; F=6.02, P<.05$, Figure $2(\mathrm{f}))$.

Interestingly, at PN45, there was no significant difference in the escape latency between group $\mathrm{T}$ and group $\mathrm{C}$ during training sessions $(P>.05$, Figure $2(\mathrm{c}))$. However, group $\mathrm{T}$ continued to spend less amount of time in searching the targeted quadrant than group $\mathrm{C}$ in the probe test $(27.80 \pm 1.51 \%$ versus $35.49 \pm 2.39 \% ; F=7.10, P<.05$, Figure 2(f)). 

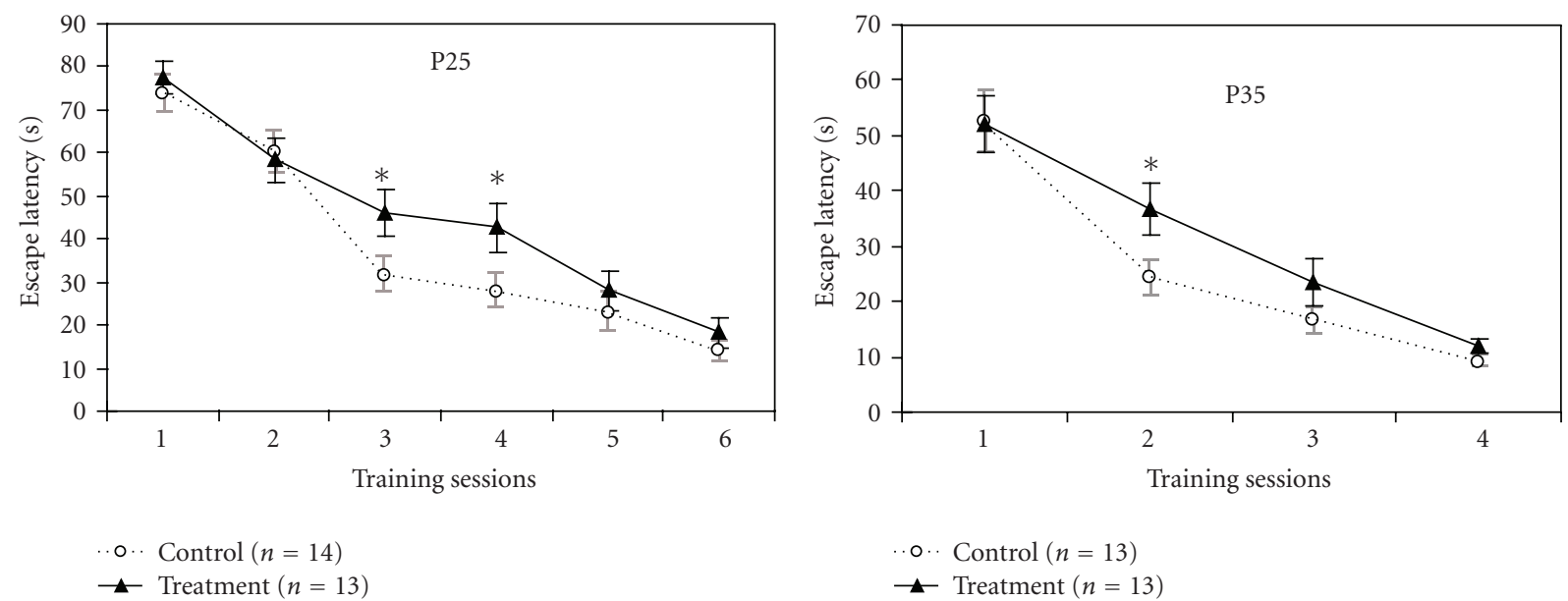

(a)

(b)
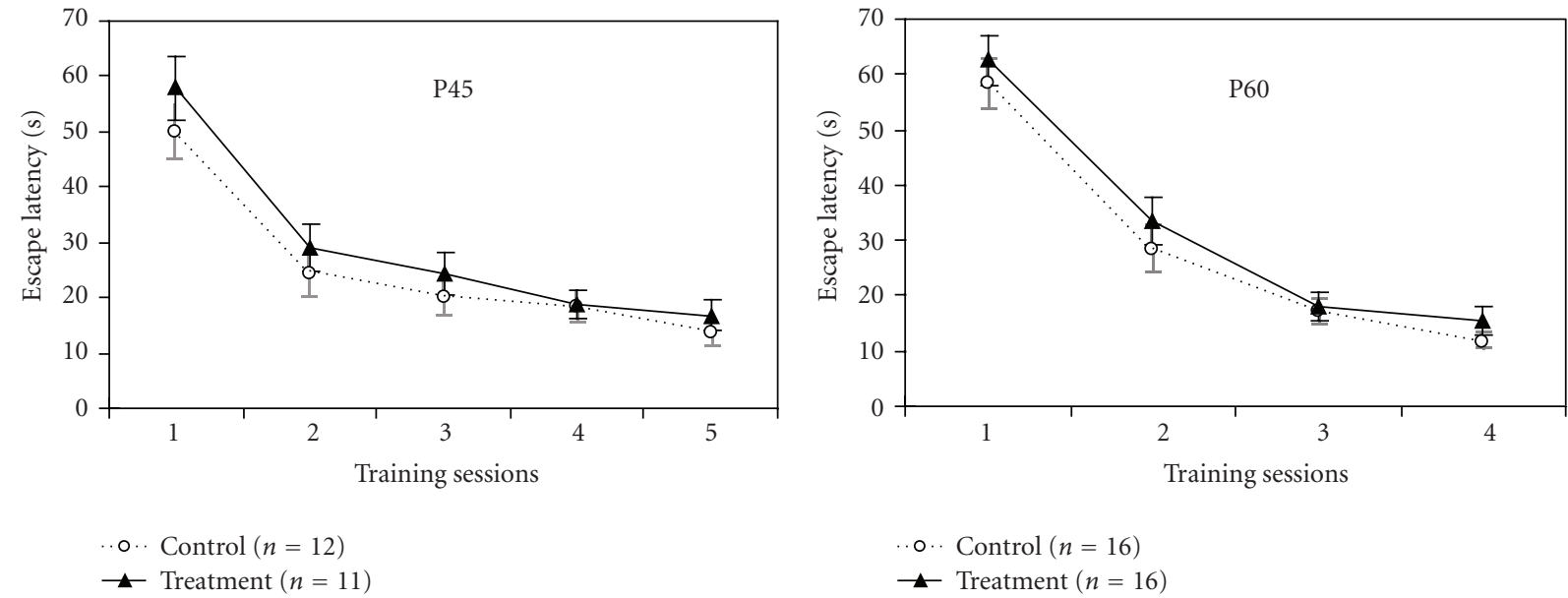

(c)
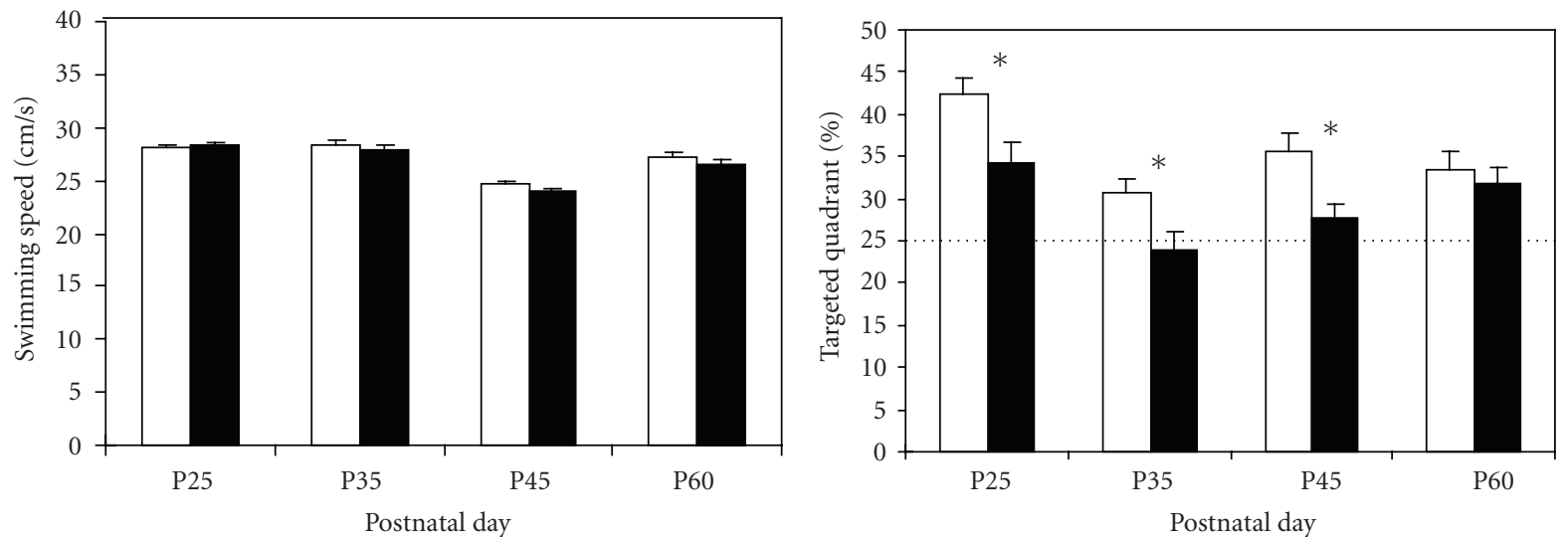

Control

Treatment

$\square$ Control

areatment

(e)

(f)

FIGURE 2: Morris water maze performance in forepaw sensorimotor deprived group (treatment) and sham-operated control group (control) at indicated age. (a) Escape latency for PN25 during training sessions, (b) PN35, (c) PN45, (d) PN60, (e) Swimming speed, and (f) The time spent in searching the targeted quadrant in the probe test for all groups. Data are presented as means \pm S.E.M. * Indicates a significant difference between groups for a given age $(P<.05)$. Number of rats $(n)$ is indicated in the figure. 
At PN60, there were no significant difference in both the escape latency during the training sessions $(P>.05$, Figure 2(d)) and the time spent in searching the targeted quadrant in the probe test $(P>.05$; Figure $2(\mathrm{f}))$ between group $\mathrm{T}$ and group $\mathrm{C}$.

Swimming speed across trials for all groups was also analyzed. There was no difference between age groups $(P>.05$, Figure 2(e)). This result indicated that forepaw sensorimotor deprivation did not affect the gross motor required for swimming. This also proved that latency to the platform was not influenced by extraneous variables such as fitness.

Taking together, these behavioral outcomes showed that the forepaw sensorimotor deprivation induced a severe delay in the maturation of spatial reference learning and memory function in rats in early life.

3.2. Synaptic Plasticity in MPP-DG Pathway. To further understand the underlying mechanism of the forepaw sensorimotor deprivation caused-impairments on learning and memory, we examined its effect on synaptic plasticity by measuring long-term potentiation in the MPP-DG pathway of hippocampal slices from young rats.

We found that both forepaw sensorimotor deprived (group T) and sham-operated control (group C) exhibited a significant degree of LTP, as measured 45 minutes following the application of the conditioning stimuli. However, there was significant difference between group $\mathrm{T}$ and group $\mathrm{C}$. Despite the high degree of LTP, group T showed significantly less LTP in comparison to group C at PN25 (157.07 $\pm 6.52 \%$ versus $188.36 \pm 6.85 \% ; F=10.95, P<.01$, Figures $3(\mathrm{a})$ and $3(\mathrm{e}))$. In addition, LTP evoked by high-frequency stimulation in group T was significantly less than that of group C at PN35 $(136.99 \pm 7.23 \%$ versus $174.21 \pm 7.65 \% ; F=12.34, P<.01$, Figures 3(b) and 3(e)). At PN45, group T continued to show significantly less LTP than group C $(143.21 \pm 6.72 \%$ versus $171.26 \pm 5.83 \% ; F=10.03, P<.01$, Figures 3(c) and $3(\mathrm{e}))$. At PN60, however, there was no significant difference between group T and group C in LTP (143.22 $\pm 6.25 \%$ versus $157.91 \pm 7.48 \% ; F=2.21, P>.05$, Figures $3(\mathrm{~d})$ and $3(\mathrm{e}))$.

Taking together, these data correlated perfectly with the behavior results suggesting that through the impairment of the NMDA receptor-mediated long-term synaptic plasticity in hippocampus, the forepaw sensorimotor deprivation induced a delay in the maturation of spatial learning and memory function of young rats.

\section{Discussion}

To date, the relationship between sensorimotor function and cognitive development in early life has been an increasing interest in neuroscience research. Accumulating data suggest that sensory input and fine motor skills in early life may facilitate the structural and functional maturation of the brain, and promote the cognitive development [11, 24, 25]. However, the impact and the underlying mechanism remain unclear. In our study here, through forepaw sensorimotor deprivation, we have systematically tested the hypothesis that forepaw sensorimotor deprivation has detrimental effects on learning and memory function in rats during their early development.

In the rat, both median and ulnar nerves are mixed nerves responsible for sensory and grip movements. The strength of the grip is controlled by the median nerve through its action on extrinsic flexor muscles responsible for the flexion of the distal phalanges. The coordination of the grip is controlled by the ulnar nerve through its action on most intrinsic muscles responsible for fine phalangeal and metacarpal movements of abduction/adduction, rotation and opposition, which optimize the application of the strength on the object. Impaired performances in the grasping test and the ulnar test observed after lesion of either median or ulnar nerve confirm the above-mentioned view [11-14].

The Morris water maze (MWM) was first described two decades ago as a device to investigate spatial learning and memory in laboratory rats. In our three-trial version of MWM, rats deprived of forepaw sensorimotor displayed a slower decrease in their escape latency during the training sessions, and in the probe test, they spent significantly less amount of time in searching the targeted quadrant at PN25, PN35. At PN45, although there was no difference in escape latency between the two groups, time spent in searching the targeted quadrant for the forepaw sensorimotor deprived group was significantly less than sham control. These results indicated that forepaw sensorimotor deprivation impaired spatial learning and memory of rats, and this deficit existed until PN45.

Synaptic plasticity is the principal means by which the nervous system adapts to the external environment. It is crucial to the development of the nervous system. The most extensively studied form of synaptic plasticity in the brain is LTP. The characteristics of LTP, including rapid formation, synapse specificity, stability, and reversibility, make it an attractive model for studying certain forms of learning and memory [26]. Among the classic trisynaptic circuit of hippocampus, MPP-DG pathway exhibits NMDA receptordependent LTP $[7,20,23,27]$. Our results showed that, forepaw sensorimotor deprivation produced a persistent change in the capacity of MPP-DG pathway to exhibit LTP until PN45 (32 days following the surgery), and this "time window" was consistent with that of spatial memory deficits. Thus, our data provide a direct physiological correlation between deficits in the memory function and impairments on the synaptic plasticity although this correlation has been suggested in previous studies $[6,7,27]$. The impairment of synaptic plasticity in MPP-DG pathway is therefore an important mechanism for the spatial memory deficits caused by forepaw sensorimotor deprivation. In addition, we previously found that synaptic plasticity in schaffer-CA1 pathway was impaired, and NMDAR1 expression level was significantly decreased after forepaw sensorimotor deprivation [15]. Because LTP in MPP-DG pathway is also NMDA receptor-dependent $[7,20,23,27]$, these data indicate that alteration of NMDA receptor subunits expression in hippocampus may also exist. 


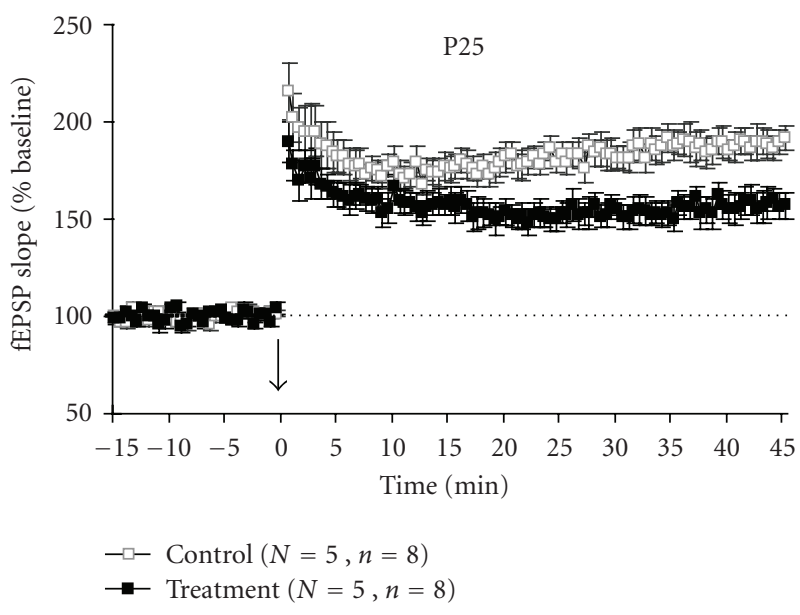

(a)

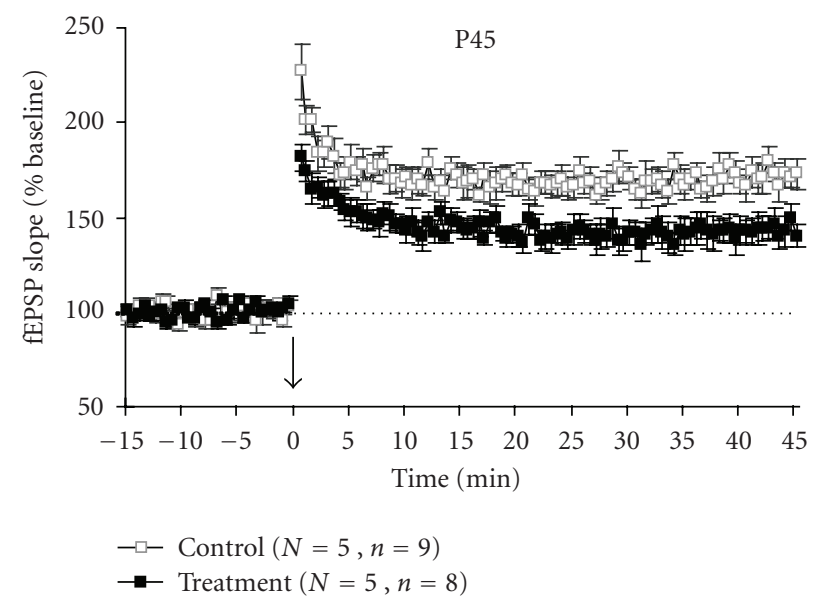

(c)

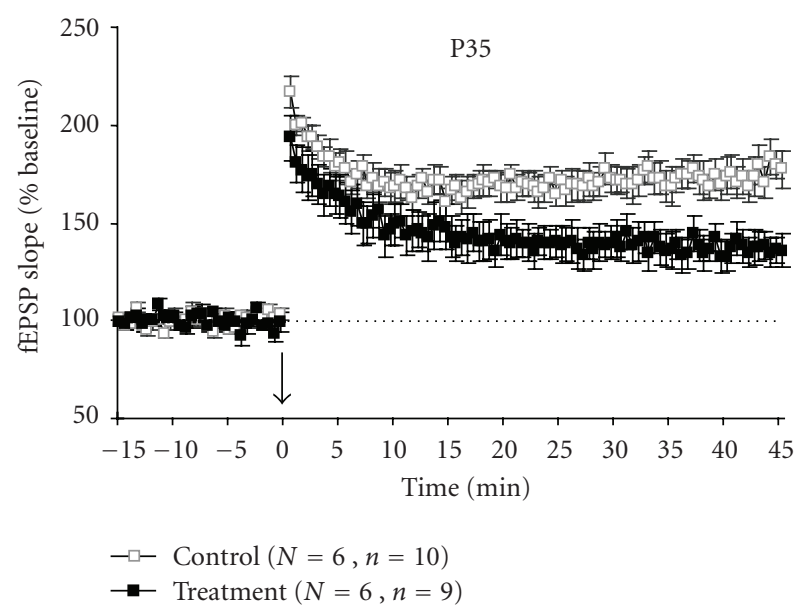

(b)

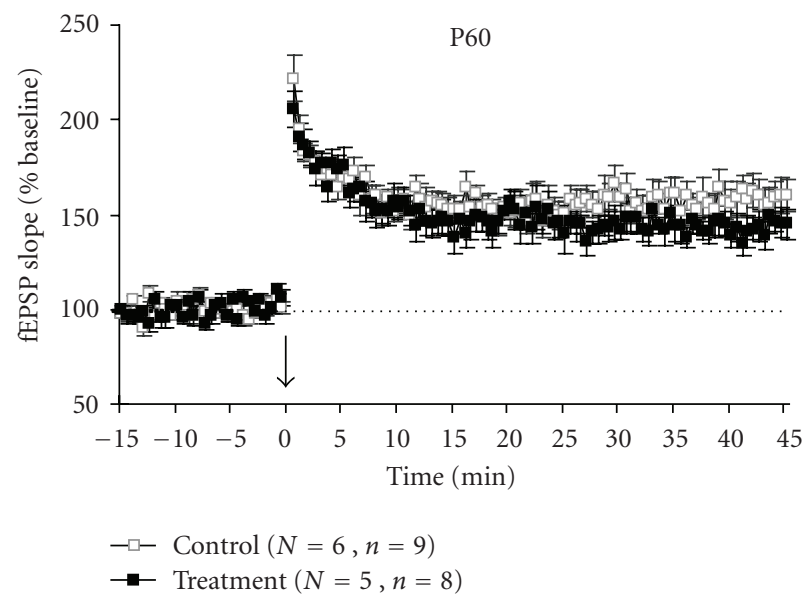

(d)

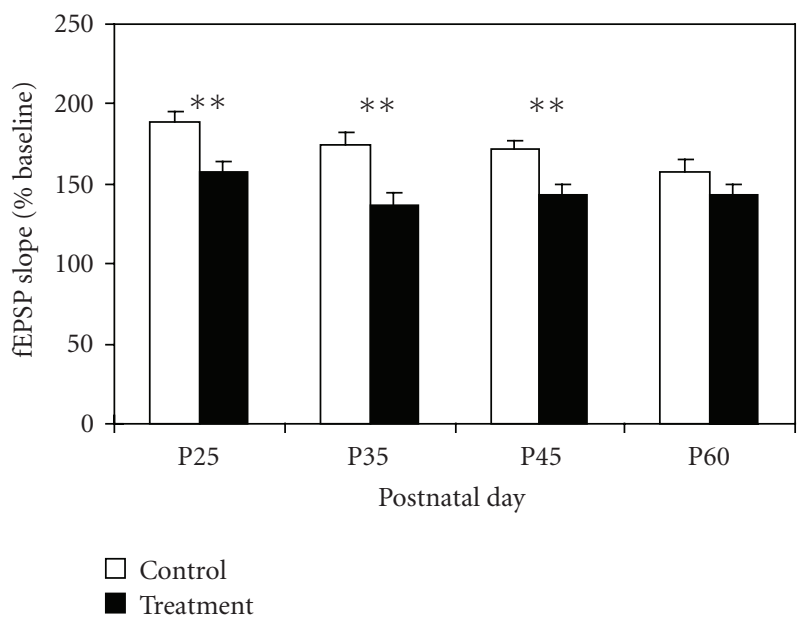

(e)

FIGURE 3: LTP induction in the medial perforant path-dentate gyrus (MPP-DG) pathway in sensorimotor deprived group (treatment) and sham-operated control group (control) at indicated age. (a) PN25, (b) PN35, (c) PN45, (d) PN60. Arrow indicates the time point when conditioning stimulation was administered. Numbers of rats $(N)$ and slices $(n)$ are indicated in all figures. (e) Summary of LTP induction at PN25, PN35, PN45, and PN60. ${ }^{* *}$ Indicates a highly significant between group difference for a given age $(P<.01)$. 
There are several possibilities for which the impairments in synaptic plasticity and spatial memory were not evident at PN60 in our study. First, the enhancement of the radial nerve function may complement the effect of removal of median and ulnar nerves because previous studies have shown that damages to median nerve in early age may result in enhanced radial nerve function. Moreover, the impulse transmission between the median nerve and the corresponding region of the brain may be replaced by that of radial nerve $[28,29]$. Second, it can be due to the brain functional reorganization. As we already know, brain plasticity is much superior in early life. Therefore, selfcompensation in the brain still experiencing maturation may remedy the impairments induced by forepaw sensorimotor deprivation.

Forepaw sensorimotor deprivation was once performed at PN18, the impairments on synaptic plasticity (in SchafferCA1 pathway) and spatial memory disappeared 17 days after the surgery [15]. However, in this study where the forepaw sensorimotor deprivation was performed at PN13, the effects on synaptic plasticity (in MPP-DG pathway) and spatial memory lasted for 32 days. This indicates that earlier deprivation of forepaw sensorimotor function can induce prolonged and more severe impairments on brain development.

There are complex interactions among learning/memory, synaptic plasticity, and neurogenesis. Hippocampus LTP is considered to be the electrophysiological basis for learning and memory $[26,30,31]$ in which new born granule cells play an important role in dentate gyrus synaptic plasticity [23], and hippocampus-dependent learning and memory as spatial reference memory is related to DG neurogenesis [32]. Many environmental factors, including physical and chemical environmental agents and social factors have been shown to influence DG neurogenesis. Behavioral rehabilitation training, enriched environment, forced or voluntary physical activity in a running wheel all have been shown to promote DG neurogenesis in rodents, facilitate induction of LTP, and enhance learning and memory performance $[7,33-$ 35]. It has been well known that fine motor training is part of rehabilitation training that facilitates recovery from injury such as cerebral ischemia and improves cognitive function [36]. Thus, forepaw sensorimotor deprivation may reduce neurogenesis level, and this in turn impairs the magnitude of hippocampal LTP and leads to spatial memory deficits as we observed. Clearly, to prove the hypothesis, further investigation is needed to determine whether neurogenesis in dentate gyrus is impaired after forepaw sensorimotor deprivation.

\section{Conclusion}

To summarize, in this study, we demonstrated that forepaw sensorimotor deprivation during early postnatal development led to transitory but severe impairments on spatial learning and memory which correlated with alterations in the synaptic plasticity in MPP-DG pathway. This provides experimental evidence supporting the relationship between cognitive development and sensorimotor function.

\section{Acknowledgments}

The authors thank the staff in Shanghai Institute of Brain Functional Genomics for their direction and assistant. This work was funded by National Natural Science Foundation of China (Grant no. 30672272), Science and Technology Commission of Shanghai Municipality (STCSM) (Grant no. 05DJ14007), Shanghai Key Laboratory of Children's Environmental Health (Grant no. 06DZ22024), Healthy Bureau of Shanghai City (Grant no. 2006-46), and Post Doctoral Funding Project of Shanghai City (Grant no. 05R214126).

\section{References}

[1] J. Piaget and B. Inhelder, La Psychologie de L'Enfant [The Psychology of the Child], Presses Universitaires de France, Paris, France, 1966.

[2] P. S. Churchland, Neurophilosophy: Toward a Unified Science of the Mind-Brain, MIT Press, Cambridge, Mass, USA, 1986.

[3] P. S. Churchland, Brain-Wise: Studies in Neurophilosophy, MIT Press, Cambridge, Mass, USA, 2002.

[4] R. Wassenberg, F. J. M. Feron, A. G. H. Kessels, et al., "Relation between cognitive and motor performance in 5- To 6-yearold children: results from a large-scale cross-sectional study," Child Development, vol. 76, no. 5, pp. 1092-1103, 2005.

[5] A. Losse, S. E. Henderson, D. Elliman, D. Hall, E. Knight, and M. Jongmans, "Clumsiness in children-do they grow out of it? a 10-year follow-up study," Developmental Medicine and Child Neurology, vol. 33, no. 1, pp. 55-68, 1991.

[6] H. Van Praag, B. R. Christie, T. J. Sejnowski, and F. H. Gage, "Running enhances neurogenesis, learning, and long-term potentiation in mice," Proceedings of the National Academy of Sciences of the United States of America, vol. 96, no. 23, pp. 13427-13431, 1999.

[7] J. Farmer, X. Zhao, H. Van Praag, K. Wodtke, F. H. Gage, and B. R. Christie, "Effects of voluntary exercise on synaptic plasticity and gene expression in the dentate gyrus of adult male Sprague-Dawley rats in vivo," Neuroscience, vol. 124, no. 1, pp. 71-79, 2004.

[8] B. R. Christie, S. E. Swann, C. J. Fox, et al., "Voluntary exercise rescues deficits in spatial memory and long-term potentiation in prenatal ethanol-exposed male rats," European Journal of Neuroscience, vol. 21, no. 6, pp. 1719-1726, 2005.

[9] R. A. Hodgson, Z. Ji, S. Standish, T. E. Boyd-Hodgson, A. K. Henderson, and R. J. Racine, "Training-induced and electrically induced potentiation in the neocortex," Neurobiology of Learning and Memory, vol. 83, no. 1, pp. 22-32, 2005.

[10] J. Biernaskie and D. Corbett, "Enriched rehabilitative training promotes improved forelimb motor function and enhanced dendritic growth after focal ischemic injury," Journal of Neuroscience, vol. 21, no. 14, pp. 5272-5280, 2001.

[11] I. E. Kudryashov and I. V. Kudryashova, "The effects of forelimb deafferentation on the post-natal development of synaptic plasticity in the hippocampus," Neuroscience and Behavioral Physiology, vol. 31, no. 3, pp. 305-310, 2001.

[12] J. A. Bertelli and J. C. Mira, "The grasping test: a simple behavioral method for objective quantitative assessment of peripheral nerve regeneration in the rat," Journal of Neuroscience Methods, vol. 58, no. 1-2, pp. 151-155, 1995.

[13] I. Papalia, P. Tos, F. Stagno d'alcontres, B. Battiston, and S. Geuna, "On the use of the grasping test in the rat median nerve 
model: a re-appraisal of its efficacy for quantitative assessment of motor function recovery," Journal of Neuroscience Methods, vol. 127, no. 1, pp. 43-47, 2003.

[14] I. Papalia, P. Tos, A. Scevola, S. Raimondo, and S. Geuna, "The ulnar test: a method for the quantitative functional assessment of posttraumatic ulnar nerve recovery in the rat," Journal of Neuroscience Methods, vol. 154, no. 1-2, pp. 198-203, 2006.

[15] F. Li, Y.-Y. Zhang, X.-M. Jing, C.-H. Yan, and X.-M. Shen, "The influence of forepaw palmar sensorimotor deprivation on learning and memory in young rats," Neuroscience Research, vol. 63, no. 1, pp. 17-23, 2009.

[16] Y. J. Li, S. L. Peng, C. Q. Wan, L. Cao, and Y. P. Li, "Chronic pain impairs spatial learning and memory ability and downregulates Bcl-2 and BDNF mRNA expression in hippocampus of neonatal rats," Zhonghua Er Ke Za Zhi, vol. 43, no. 6, pp. 444-448, 2005.

[17] R. D’hooge and P. P. De Deyn, “Applications of the Morris water maze in the study of learning and memory," Brain Research Reviews, vol. 36, no. 1, pp. 60-90, 2001.

[18] X. Cao, Z. Cui, R. Feng, et al., "Maintenance of superior learning and memory function in NR2B transgenic mice during ageing," European Journal of Neuroscience, vol. 25, no. 6, pp. 1815-1822, 2007.

[19] Y.-P. Tang, E. Shimizu, G. R. Dube, et al., "Genetic enhancement of learning and memory in mice," Nature, vol. 401, no. 6748, pp. 63-69, 1999.

[20] B. L. McNaughton and C. A. Barnes, "Physiological identification and analysis of dentate granule cell responses to stimulation of the medial and lateral perforant pathways in the rat," The Journal of Comparative Neurology, vol. 175, no. 4, pp. 439-453, 1977.

[21] B. R. Christie and W. C. Abraham, "Priming of associative long-term depression in the dentate gyrus by $\theta$ frequency synaptic activity," Neuron, vol. 9, no. 1, pp. 79-84, 1992.

[22] B. R. Christie and W. C. Abraham, "NMDA-dependent heterosynaptic long-term depression in the dentate gyrus of anaesthetized rats," Synapse, vol. 10, no. 1, pp. 1-6, 1992.

[23] J. S. Snyder, N. Kee, and J. M. Wojtowicz, "Effects of adult neurogenesis on synaptic plasticity in the rat dentate gyrus," Journal of Neurophysiology, vol. 85, no. 6, pp. 2423-2431, 2001.

[24] I. E. Kudryashov and I. V. Kudryashova, "Age-related changes in the characteristics of evoked responses in field CA1 in rat hippocampus slices after deafferentation of the forelimb," Neuroscience and Behavioral Physiology, vol. 35, no. 2, pp. 139146, 2005.

[25] F. Wurm, S. Keiner, A. Kunze, O. W. Witte, and C. Redecker, "Effects of skilled forelimb training on hippocampal neurogenesis and spatial learning after focal cortical infarcts in the adult rat brain," Stroke, vol. 38, no. 10, pp. 2833-2840, 2007.

[26] T. V. P. Bliss and G. L. Collingridge, "A synaptic model of memory: long-term potentiation in the hippocampus," Nature, vol. 361, no. 6407, pp. 31-39, 1993.

[27] G. Richter-Levin, L. Canevari, and T. V. P. Bliss, "Long-term potentiation and glutamate release in the dentate gyrus: links to spatial learning," Behavioural Brain Research, vol. 66, no. 12, pp. 37-40, 1995.

[28] P. E. Garraghty, L. L. Arnold, C. L. Wellman, and T. M. Mowery, "Receptor autoradiographic correlates of deafferentationinduced reorganization in adult primate somatosensory cortex," The Journal of Comparative Neurology, vol. 497, no. 4, pp. 636-645, 2006.

[29] G. Lundborg, "Richard P. Bunge memorial lecture. Nerve injury and repair-a challenge to the plastic brain," Journal of the Peripheral Nervous System, vol. 8, no. 4, pp. 209-226, 2003.
[30] R. G. M. Morris, R. F. Halliwell, and N. Bowery, "Synaptic plasticity and learning II: do different kinds of plasticity underlie different kinds of learning?" Neuropsychologia, vol. 27, no. 1, pp. 41-59, 1989.

[31] S. Maren and M. Baudry, "Properties and mechanisms of longterm synaptic plasticity in the mammalian brain: relationships to learning and memory," Neurobiology of Learning and Memory, vol. 63, no. 1, pp. 1-18, 1995.

[32] E. Drapeau, W. Mayo, C. Aurousseau, M. Le Moal, P.-V. Piazza, and D. N. Abrous, "Spatial memory performances of aged rats in the water maze predict levels of hippocampal neurogenesis," Proceedings of the National Academy of Sciences of the United States of America, vol. 100, no. 24, pp. 14385-14390, 2003.

[33] H. Van Praag, G. Kempermann, and F. H. Gage, "Running increases cell proliferation and neurogenesis in the adult mouse dentate gyrus," Nature Neuroscience, vol. 2, no. 3, pp. 266-270, 1999.

[34] W. P. Xu, L. D. Shan, S. Gong, et al., "Forced running enhances neurogenesis in the hippocampal dentate gyrus of adult rats and improves learning ability," Sheng Li Xue Bao, vol. 58, no. 5, pp. 415-420, 2006.

[35] T. L. Briones, E. Suh, H. Hattar, and M. Wadowska, "Dentate gyrus neurogenesis after cerebral ischemia and behavioral training," Biological Research for Nursing, vol. 6, no. 3, pp. 167179, 2005.

[36] P. Tomlin, M. Clarke, G. Robinson, and J. Roach, "Rehabilitation in severe head injury in children: outcome and provision of care," Developmental Medicine and Child Neurology, vol. 44, no. 12, pp. 828-837, 2002. 

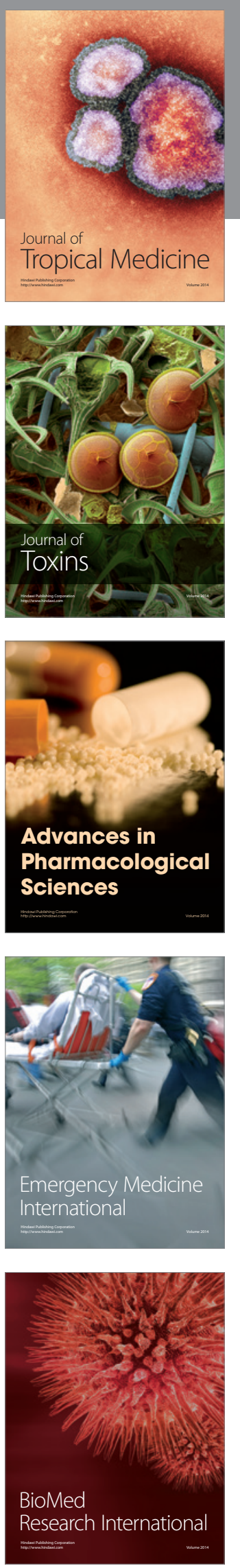
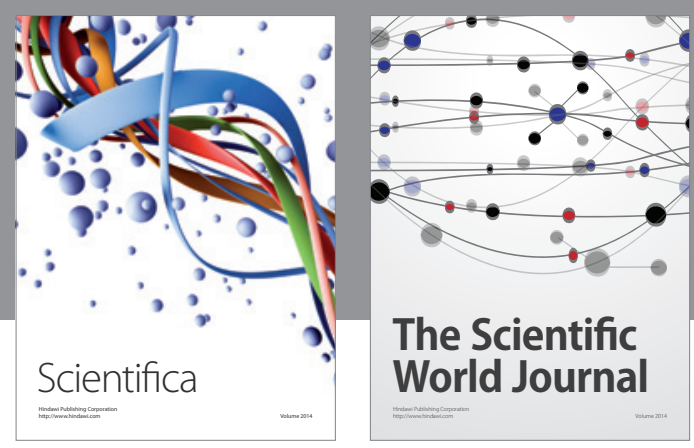

The Scientific World Journal
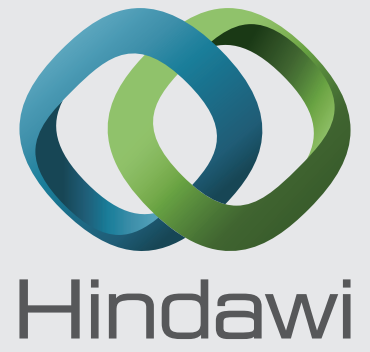

Submit your manuscripts at

http://www.hindawi.com
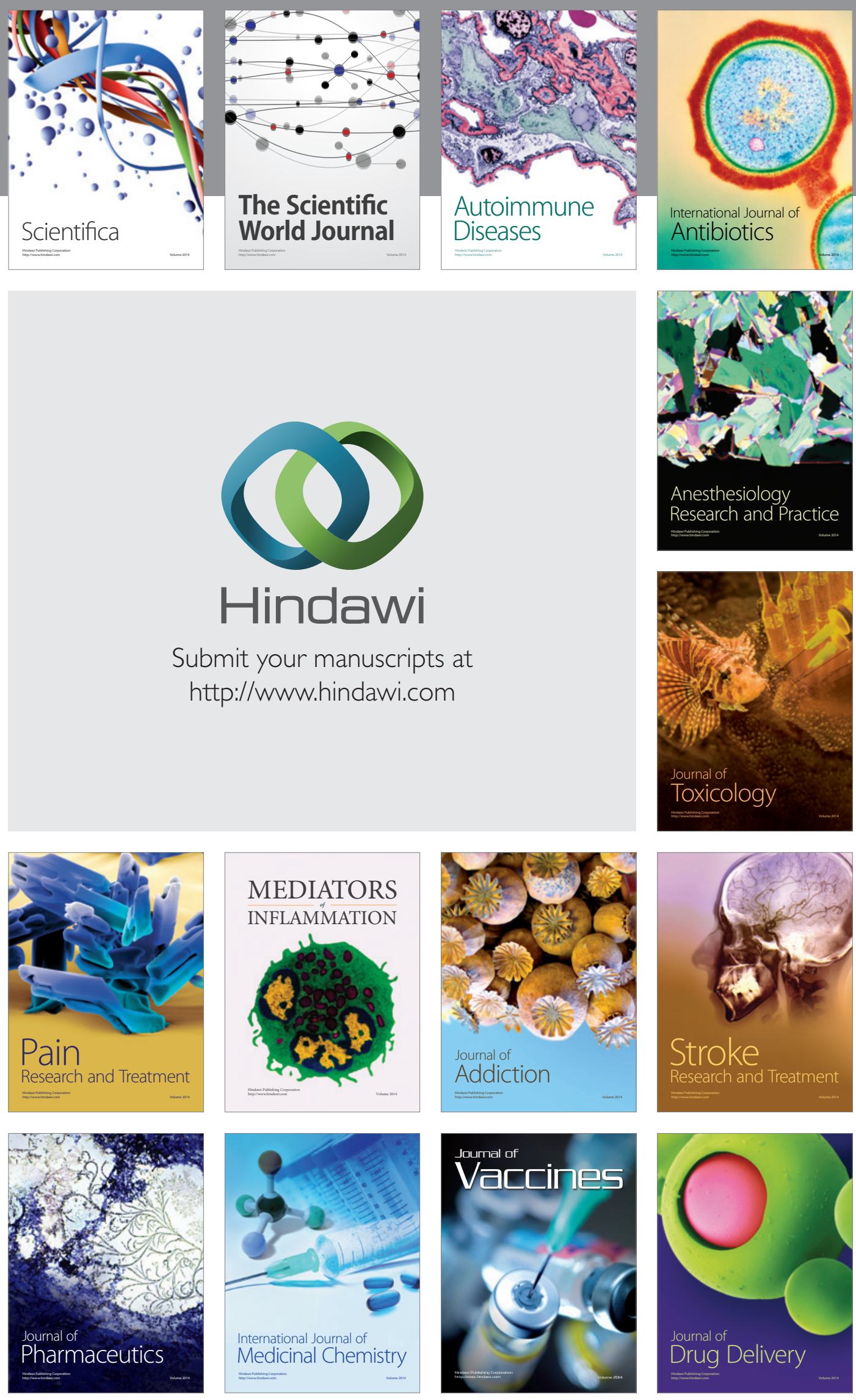